\title{
Variational Chaos Indicators: Application to the Restricted Three-Body Problem
}

\author{
Alexey M. Koksin and Vladimir A. Shefer \\ Tomsk State University, 634050 Tomsk, Russia \\ email: shefer@niipmm.tsu.ru
}

\begin{abstract}
A comparison of several known dynamical indicators of chaos based on the numerical integration of differential variational equations is performed. The comparison is implemented on the examples of studying dynamics in the planar circular restricted three-body problem.
\end{abstract}

Keywords. celestial mechanics, methods: n-body simulations; methods: numerical; instabilities

\section{Introduction}

The classical method for distinguishing between regular and chaotic motions in dynamical systems is the calculation of the maximal Lyapunov Characteristic Number (mLCN). For a continuous dynamical system with $n$ degrees of freedom the mLCN is defined as

$$
\operatorname{mLCN} \equiv \sigma=\lim _{t \rightarrow \infty}(\ln \delta / t)
$$

using the solution of the system of motion equations and variational equations:

$$
\dot{\mathbf{x}}=\mathbf{f}(\mathbf{x}(t)), \quad \dot{\boldsymbol{\delta}}=\frac{\partial \mathbf{f}}{\partial \mathbf{x}}(\mathbf{x}(t)) \boldsymbol{\delta} ; \quad \mathbf{x}, \boldsymbol{\delta} \in R^{2 n}, \quad t \in R .
$$

Here, $t$ is the independent variable (time), the vector $\mathbf{x}(t)$ sets a trajectory in the phase space of the dynamical system; $\mathbf{x}_{0} \equiv \mathbf{x}\left(t_{0}\right), t_{0}=0$ are the initial conditions for the equations of motion, $\boldsymbol{\delta}(t)$ is the tangent vector determining the evolution of initial unit deviation vector relative to the trajectory $\mathbf{x}(t) ; \delta \equiv\|\boldsymbol{\delta}(t)\|$.

Usually the convergence of $\ln \delta / t$ to $\sigma$ is slow, therefore the reliable estimation of the mLCN requires a long calculation time. It stimulated the emergence of so-called fast Lyapunov indicators allowing to study the phase space on relatively short time intervals.

The aim of this research was to compare some often-used fast chaos indicators (hereafter CIs) on the examples of their working in the case of the dynamical system specified by the equations of the planar circular restricted three-body problem (Szebehely 1967).

\section{Numerical Experiments and Results}

We selected the following chaos indicators: the Fast Lyapunov Indicator (FLI, Froeschlé et al. 1997), the Orthogonal Fast Lyapunov Indicator (OFLI, Fouchard et al. 2002), the Mean Exponential Growth factor of Nearby Orbits (MEGNO, Cincotta \& Simo 2000), the Smaller Alignment Index (SALI, Skokos 2001), and the Average Power Law Exponent (APLE, Lukes-Gerakopoulos et al. 2008). Specific applications of some listed CIs to the circular restricted three-body problem were discussed, for example, in (Fouchard et al. 2002; Frouard et al. 2008; Morais \& Giuppone 2012).

We used also the Orthogonal Mean Exponential Growth factor of Nearby Orbits (OMEGNO) introduced by us (Shefer \& Koksin 2013). It is determined by

$$
\operatorname{OMEGNO}(t)=2(\theta-\zeta) / t,
$$


where $\theta$ and $\zeta$ are calculated by integrating the differential equations

$$
\dot{\theta}=\ln \delta_{\perp}, \quad \dot{\zeta}=\theta / t
$$

simultaneously with the equations (1.1). We use $\delta_{\perp}=\left\|\boldsymbol{\delta}_{\perp}(t)\right\|$, where $\boldsymbol{\delta}_{\perp}(t)$ is the vector component of $\boldsymbol{\delta}(t)$ orthogonal to the direction of the phase flow. The initial conditions for $(2.2)$ are as follows: $\theta(0)=0, \zeta(0)=0$. At $t=0$ the right-hand side of the second equation of system (2.2) is taken equal to zero. OMEGNO $(t) \rightarrow 0$ with $t \rightarrow \infty$ for any stable periodic orbit. Thus, this MEGNO's modification gives the indicator, which makes it possible to separate periodicity in the regular component of the phase space.

The problem considered in this work describes the motion of a massless body $P$ perturbed by two massive bodies $P_{1}$ and $P_{2}$ (called primaries) with respective masses $1-\mu$ and $\mu(\mu \leqslant 0.5)$ moving around their barycenter $O$. The length unit is such that the distance between the primaries is unity, and the time unit $\tau$ is such that the orbital period of the primaries equals $2 \pi \tau$.

In the rotating frame $x O y$, in which $P_{1}$ and $P_{2}$ are located at points with coordinates $(-\mu, 0)$ and $(1-\mu, 0)$, respectively, the equations of motion of $P$ are written in the form $\ddot{x}=2 \dot{y}+x-(1-\mu)(x+\mu) / r_{1}^{3}-\mu(x-1+\mu) / r_{2}^{3}, \quad \ddot{y}=-2 \dot{x}+y-(1-\mu) y / r_{1}^{3}-\mu y / r_{2}^{3}$, where $r_{1}^{2}=(x+\mu)^{2}+y^{2}$ and $r_{2}^{2}=(x-1+\mu)^{2}+y^{2}$.

We accepted that the primaries have equal masses $(\mu=0.5)$, and the Jacobi integral is equal to 4 .

For the initial vector $\mathbf{x}_{0} \equiv\left(x_{0}, \dot{x_{0}}, y_{0}, \dot{y_{0}}\right)$ we took the set of 961 values, where $x_{0}$ coordinate is uniformly distributed in the interval $[-0.49,-0.01]$ with the step of size $0.0005, \dot{x_{0}}=0, y_{0}=0$, and $\dot{y_{0}}>0$ is determined from the Jacobi integral.

As a method of numerical integration of differential equations the Dormand-Prince algorithm of the 8(7)th order (Hairer et al. 1987) was chosen.

With above-mentioned initial conditions we have calculated the values of the CIs. The final values on the time $t=10000 \tau$ are represented graphically (Fig. 1).

In all the figures obtained the chaotic motion appears separated from the regular resonant one as a whole. But the MEGNO and the OMEGNO separate these motions most clearly because these indicators have the universal threshold value $(\cong 2)$. The FLI, the OFLI, and the SALI have not reference values clearly discriminating the regular orbits from the weak chaotic ones.
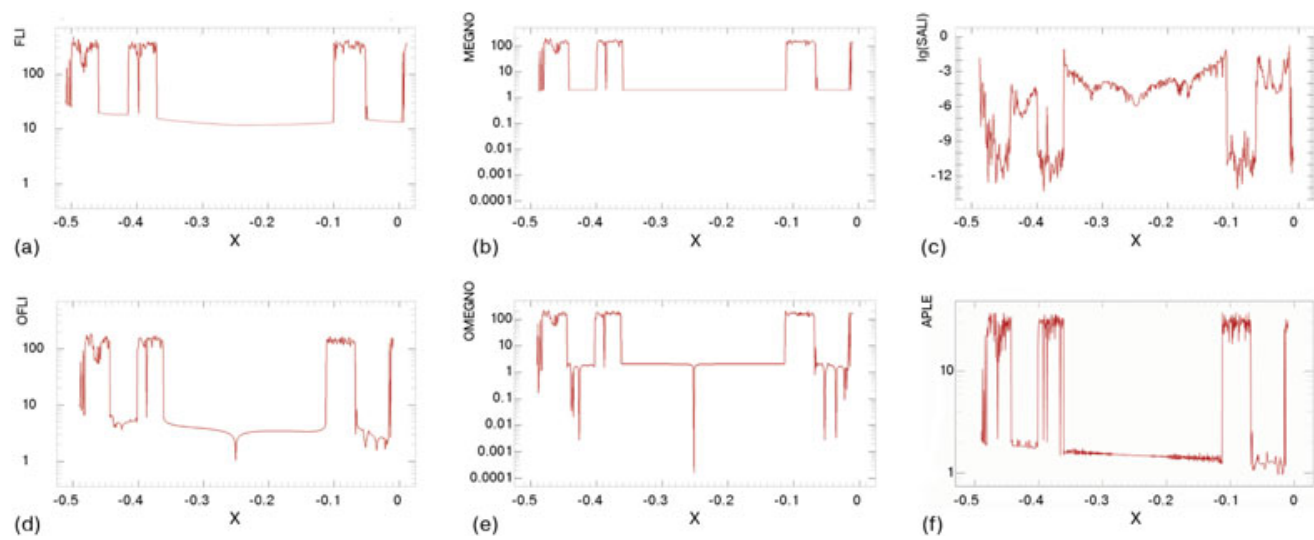

Figure 1. Final values of the CIs for an integration time of $10000 \tau$ : (a) FLI, (b) MEGNO, (c) SALI, (d) OFLI, (e) OMEGNO, (f) APLE. 
There is a difference between the values of the FLI, the OFLI, the SALI, and the APLE found for $x_{0}$ in $[-0.44,-0.40]$ and $[-0.065,-0.015]$. As both intervals correspond to the same set of orbits, this difference can be explained only by the relative positions of the initial vectors $\mathbf{x}_{0}$ and $\boldsymbol{\delta}_{0}$ (analogous conclusions were made by Fouchard et al. (2002)).

A comparison shows that on the curves of the OFLI and the OMEGNO are several minima, which are not presented on the curves of the FLI and the MEGNO. These minima refer to the presence of periodic orbits in the neighbourhood of the corresponding initial conditions. The final values of the SALI and the APLE demonstrate minima in the regular motion areas too, but some of them are not related with the periodic component of the motion. By the final values of the FLI and the MEGNO it is not possible to identify a fine structure of the regular component of the motion.

For effective study of large sets of initial conditions with the help of the CIs' final values it is important to know the reliability on the CIs' thresholds that make a confident distinction between chaotic and regular motions.

We took the same initial conditions as in the previous experiment and identified the chaotic orbits by the Poincaré surface of section (there are found 263 chaotic orbits, i.e. $27,4 \%$ ). The indicators we chose have the theoretical estimations of their thresholds (time-dependent or time-independent). We splitted the full time of integration $(10000 \tau)$ on 20 sub-intervals per $500 \tau$ for each. We have computed the number of chaotic orbits in each sub-interval with each indicator on the basis of it threshold. We estimated the reliability on the corresponding threshold by comparing this number with the true count of chaotic orbits.

The thresholds associated with the FLI and the APLE define all 961 orbits as chaotic ones for the complete time interval. The thresholds for the MEGNO and the SALI give the number of chaotic orbits increased approximately 2.5 times. So they are inefficient too.

The thresholds for the orthogonal indicators are very close to the values allowing to achieve a stable fraction of the chaotic component, close to the true fraction.

Consequently, the thresholds' estimations for the FLI, the APLE, the MEGNO, and the SALI are not reliable and they need an empirical adjustment. The thresholds for the OFLI and the OMEGNO practically do not require any corrections.

To trace the evolution of the CIs' values with time for different types of motion, we considered four representative orbits with the following initial conditions for $x_{0}:-0.25065550$ $\left(O_{1}\right),-0.35\left(O_{2}\right),-0.28\left(O_{3}\right),-0.08\left(O_{4}\right)$. The values of $\dot{x_{0}}, y_{0}$, and $\dot{y_{0}}$ are the same as in the cited above set of initial conditions. The orbit $O_{1}$ is a periodic orbit, $O_{2}$ and $O_{3}$ are resonance orbits from the $O_{1}$ 's neighbourhood, $O_{4}$ belongs to chaotic orbits. The initial conditions for $O_{1}, O_{3}$, and $O_{4}$ were taken from (Fouchard et al. 2002). The variations of the CIs' values for four selected orbits in the time interval $10000 \tau$ are showed graphically in a logarithmic time scale (Fig. 2).

In this numerical experiment all the CIs clearly separate the regular resonance motion from the strong chaotic one.

In regard to separation of different levels of the regular motion, here the results of the CIs' working can be described as following.

The FLI, the MEGNO, and the APLE for the resonance libration orbits detect their stable quasi-periodic nature very quickly and show a power of their proximity to $O_{1}$. But in this case we can not say uniquely that $O_{1}$ is a periodic orbit. The rest of the CIs not only separate the quasi-periodic orbits between themselves, but they detect the periodic orbit. As this takes place, the closer the quasi-periodic orbit to the periodic one the later their essential distinction is detected. 

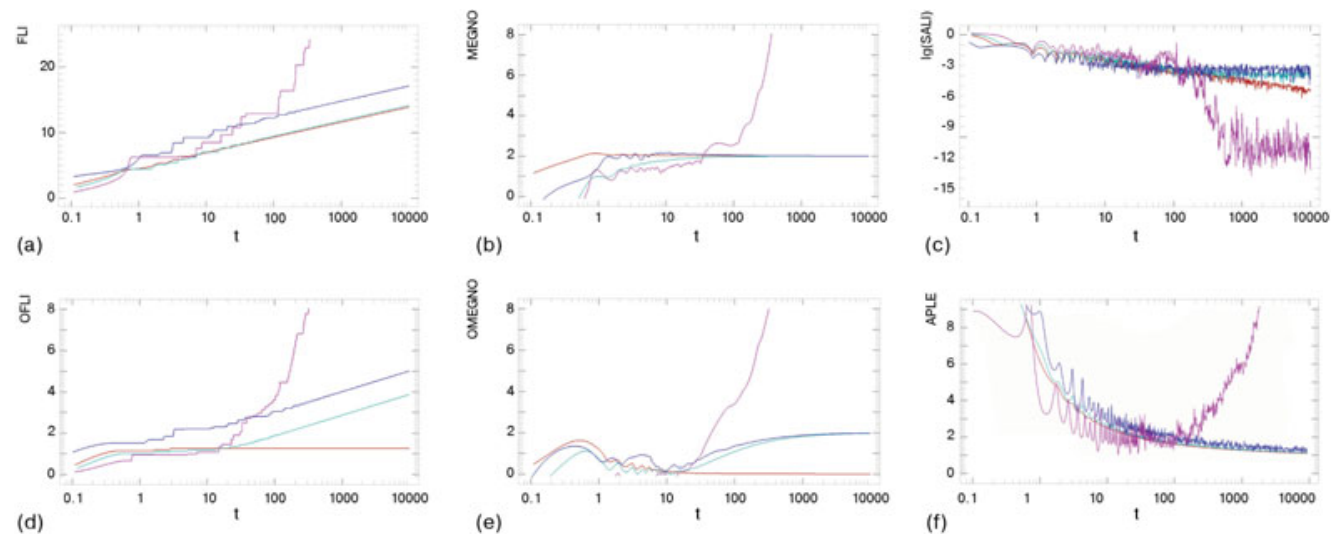

Figure 2. Evolution of the CIs with time for the four representative orbits $O_{1}$ (the red line), $\mathrm{O}_{2}$ (the light-blue line), $\mathrm{O}_{3}$ (the dark-blue line), and $\mathrm{O}_{4}$ (the purple line): (a) FLI, (b) MEGNO, (c) SALI, (d) OFLI, (e) OMEGNO, (f) APLE.

For each quasi-periodic orbit the OFLI temporarily takes a constant value (the plateau in figure) but later it grows linearly with the same slope. In this case the length of the plateau increases with approaching the quasi-periodic orbit to the periodic one. Hence at the end of the selected integration interval the OFLI's value for the orbit more close to the periodic one is less compared with the OFLI's value for the more distant orbit.

The OMEGNO's values for the quasi-periodic orbits in very short time confidently go away from zero approaching the value 2 from below. The farther the quasi-periodic orbit is from the periodic one the earlier the OMEGNO's values fall into the nearest neighbourhood of 2 .

The APLE's values for $O_{1}, O_{2}$, and $O_{3}$ approache unity from above with time. The closer the quasi-periodic orbit to the periodic one the closer the APLE's values to unity.

The function $\lg (\mathrm{SALI})$ for the quasi-periodic orbits reaches different constant values. Here, the closer is the quasi-periodic orbit to the periodic one the lower is the plateau and the later is reached this plateau.

Thus, the OFLI, the OMEGNO, and the SALI allow to detect and locate periodic orbits. But the OMEGNO is only CI from these having the universal reference value $(\cong 0)$ for periodic motion. The OFLI and the SALI give only relative results, which require calibration with a known reference orbit.

The work was supported by the Ministry of Education and Science of the Russian Federation, project no. 2014/223(1766).

\section{References}

Cincotta, P. M., Simo, C. 2000, Astron. Astrophys. Suppl. Ser., 147, 205

Fouchard, M., Lega, E., Froeschlé, Ch., \& Froeschlé, C. 2002, Celest. Mech. Dyn. Astr., 83, 205

Froeschlé, C., Lega, E., \& Gonczi, R. 1997, Celest. Mech. Dyn. Astr., 67, 41

Frouard, J., Fouchard, M., \& Vienne, A. 2008, SF2A-2008, 121

Hairer, E., Norsett, S. P., \& Wanner, G. 1987, Solving Ordinary Differential Equations. Nonstiff Problems (Berlin, Heidelberg: Springer-Verlag)

Lukes-Gerakopoulos, G., Voglis, N., \& Efthymiopoulos, C. 2008, Physica A, 387, 1907

Morais, M. H. M. \& Giuppone, C. A. 2012, MNRAS, 424, 52

Shefer, V. A. \& Koksin, A. M. 2013, Izv. Vusov. Fizika, 56, No.6/3, 256

Skokos, Ch. 2001, J. Phys. A, 34, 10029

Szebehely, V. 1967, Theory of Orbits (New York: Academic Press) 